\title{
The infrared spectrum of the benzene-Ar cation
}

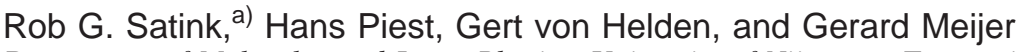 \\ Department of Molecular and Laser Physics, University of Nijmegen, Toernooiveld, \\ NL-6525 ED Nijmegen, The Netherlands and FOM-Institute for Plasma Physics Rijnhuizen, \\ Edisonbaan 14, NL-3430 BE Nieuwegein, The Netherlands
}

(Received 10 September 1999; accepted 21 October 1999)

\begin{abstract}
The infrared (IR) absorption spectra of the jet-cooled $\mathrm{C}_{6} \mathrm{H}_{6}$ and $\mathrm{C}_{6} \mathrm{D}_{6}$ cations, complexed with $\mathrm{Ar}$, are measured throughout the $450-1500 \mathrm{~cm}^{-1}$ region via IR-laser-induced vibrational dissociation spectroscopy. The IR spectrum of the $\mathrm{C}_{6} \mathrm{H}_{6}-\mathrm{Ar}$ cation is dominated by a Fermi resonance between the IR active $\nu_{11}$ mode and two components of the combination mode of the lowest frequency modes $\nu_{6}$ and $\nu_{16}$. A stringent upper limit of $316 \mathrm{~cm}^{-1}$ is found for the value of the dissociation limit $D_{0}$ of the neutral $\mathrm{C}_{6} \mathrm{D}_{6}-\mathrm{Ar}$ complex. (C) 1999 American Institute of Physics.

[S0021-9606(99)01548-2]
\end{abstract}

The benzene cation with its doubly degenerate $\widetilde{X}^{2} E_{1 g}$ electronic ground state is arguably the most prototypical example of a Jahn-Teller distorted system. According to Jahn and Teller $^{1}$ the coupling of nuclear motion with the electronic degeneracy leads to a distortion of the molecule along certain normal coordinates so that the potential minimum is no longer at the symmetrical position. Consequently, there has been considerable interest to unravel the vibrational properties and to deduce information on this Jahn-Teller distorted structure for the benzene cation. ${ }^{2}$ For many of the halogenated benzenoid cations gas-phase laser-inducedfluorescence (LIF) studies have been applied and have yielded a wealth of information and improved understanding on the Jahn-Teller effect as such, as in these studies the Jahn-Teller active modes are directly accessible. ${ }^{3,4}$ Its low fluorescence quantum yield has prohibited such gas-phase LIF studies on the benzene cation. Photoelectron spectroscopy ${ }^{5}$ and threshold-electron (zero electron kinetic energy, ZEKE) ${ }^{6-8}$ and threshold-ion detection (mass analyzed threshold ionization, MATI) schemes ${ }^{9-11}$ have been successfully used, however, to measure and assign several vibrational modes in the benzene cations.

In this communication we report the first gas-phase IR absorption spectra of the $\mathrm{C}_{6} \mathrm{H}_{6}$ and $\mathrm{C}_{6} \mathrm{D}_{6}$ cations, complexed with Ar, throughout the $450-1500 \mathrm{~cm}^{-1}$ region. The spectra are measured via IR-laser-induced vibrational dissociation of the laser prepared cationic van der Waals complexes. The experimental detection scheme also allows one to determine an upper limit for the dissociation energy of these weakly bonded complexes. As the rare gas atom in the ionic complex is located above the plane of the benzene molecule, on the symmetry axis, ${ }^{12}$ the van der Waals complex retains a sufficiently high symmetry to also be Jahn-Teller distorted, and the IR spectrum of the ionic complex is expected to resemble closely that of the bare benzene cation. This is substantiated by the good agreement between the observed

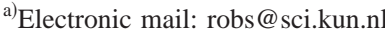

MATI spectra for bare benzene and those of its argon complexes. ${ }^{10}$

The experimental setup and the detection method that is used are similar to those described previously in our work reporting on the IR spectra of aniline-Ar, ${ }^{13}$ naphthaleneAr, ${ }^{14}$ and pyrazine-Ar, ${ }^{15}$ and are only briefly given here.

The experiments are performed using the free electron laser for infrared experiments (FELIX) ${ }^{16}$ as a source of intense pulsed infrared radiation. FELIX produces IR radiation that is continuously tunable over the $40-2000 \mathrm{~cm}^{-1}$ range. The light output consists of macropulses of about $4 \mu \mathrm{s} \mathrm{du}-$ ration containing up to $100 \mathrm{~mJ}$ of energy. Each macropulse consists of a train of micropulses that are $0.3-5 \mathrm{ps}$ long and $1 \mathrm{~ns}$ apart. In the present experiments, the macropulse repetition rate is $10 \mathrm{~Hz}$, and the bandwidth of the laser is typically set to $0.5 \%-1.0 \%$ of the central frequency. In the molecular beam apparatus, a pulsed valve backed with 2 bar Ar premixed with a fraction of a percent of $\mathrm{C}_{6} \mathrm{H}_{6}\left(\mathrm{C}_{6} \mathrm{D}_{6}\right)$, releases gas pulses of typically $50 \mu$ s duration. About $4 \mathrm{~cm}$ downstream, the molecular beam is skimmed upon entering a differentially pumped linear time-of-flight (TOF) mass spectrometer. The molecules in the beam interact with incoming UV laser beams as well as with the collimated FELIX beam (approximately 2-3 $\mathrm{mm}$ diameter) at the crossing point of the mutually perpendicular molecular beam axis, laser beam axis, and TOF-tube axis. A scheme of the experimental detection method that is used is given in Fig. 1. The van der Waals complexes under study are ionized via two-color $\left(1+1^{\prime}\right)$-resonance enhanced multiphoton ionization (REMPI) via the $6^{1}\left(S_{1}\right)$ state under field-free conditions, using the appropriate UV laser wavelengths. ${ }^{12,17}$ The energy of the ionizing photon is chosen such as to bring the van der Waals complex just barely above the ionization threshold. With an excess energy of less than $10 \mathrm{~cm}^{-1}$, even the lowest van der Waals mode of the complex cannot be excited. ${ }^{18}$ Therefore, ions will only be prepared in the ground vibrational level, and will be about as cold as the neutral benzene-Ar complexes that they originate from. Shortly after production, the complex ions are irradiated with the macropulse of FELIX. The maximum energy density of FELIX used in these ex- 


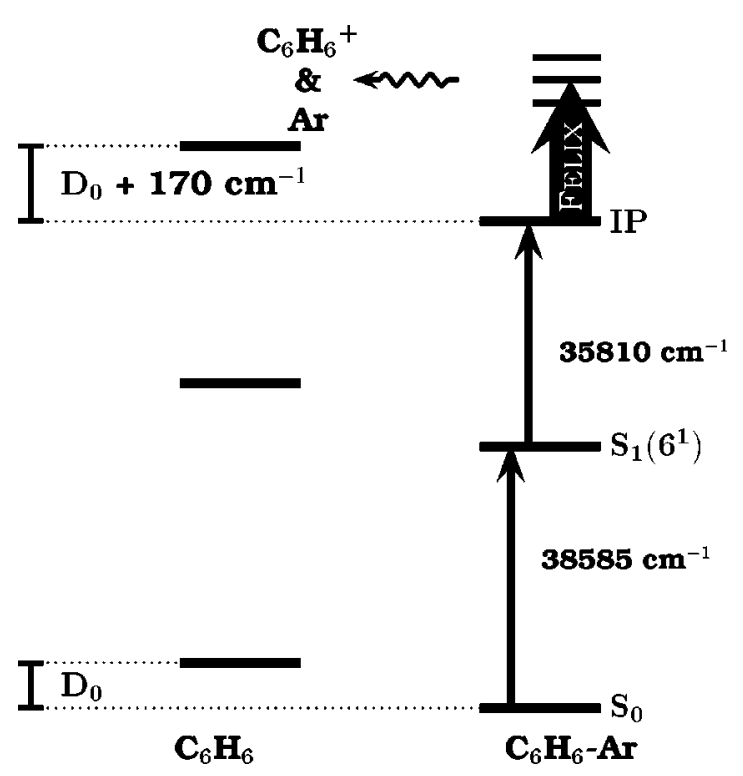

FIG. 1. Energy-level scheme for $\mathrm{C}_{6} \mathrm{H}_{6}$ and for $\mathrm{C}_{6} \mathrm{H}_{6}-\mathrm{Ar}$, indicating the experimental method used to record the IR spectrum of the complex cation.

periments is about $0.2 \mathrm{~J} / \mathrm{cm}^{2}$, which is reduced by fixedvalue attenuators if required. Several microseconds after the end of the FELIX macropulse, the ions in the interaction region are pulse extracted and accelerated to a multichannel plate (MCP) detector, yielding TOF mass spectra. If a vibrational transition is induced in the complex cation, vibrational dissociation can occur, leading to the appearance of benzene cations against zero background. The IR absorption spectrum of the internally cold benzene-Ar complex ion is measured via recording of the ratio of the benzene cation signal intensity to the sum of the benzene-Ar cation and benzene cation intensity (corrected for IR-laser intensity fluctuations) as a function of IR-laser frequency.

In the upper part of Fig. 2 the observed IR absorption spectrum of the $\mathrm{C}_{6} \mathrm{H}_{6}-\mathrm{Ar}$ cation as recorded via the scheme depicted in Fig. 1 is shown. At least 12 IR transitions can be identified in the $450-1500 \mathrm{~cm}^{-1}$ region. The spectrum is dominated by intense IR transitions in the $600-700 \mathrm{~cm}^{-1}$ region. The linewidth of the individual transitions observed in the spectrum is mainly determined by the linewidth of the IR laser. In the lower part of Fig. 2 the observed IR absorption spectrum of the deuterated ion is shown, in which 14 peaks are identified and which appears strikingly different from the observed spectrum of its hydrogen-containing counterpart. As we are measuring the IR spectrum of the van der Waals complex, it can never be excluded that van der Waals sidebands appear in the IR spectrum of the complex, but such sidebands are expected to be weak relative to the fundamental transitions. Moreover, in the case of $\mathrm{C}_{6} \mathrm{H}_{6}$ a very similar IR spectrum, only with a slightly inferior signal-tonoise ratio, has been recorded via dissociation spectroscopy on the $\mathrm{C}_{6} \mathrm{H}_{6}-\mathrm{Kr}$ complex. We can therefore be confident that the spectra shown in Fig. 2 are rather close both in line position (within a few $\mathrm{cm}^{-1}$ ) and in relative line intensity to the "true" IR absorption spectra of the bare benzene cations.

We will compare the observed spectra to the IR spectra that are expected for the bare benzene cations. The vibrational modes of benzene, with the molecular $D_{6 h}$ symmetry,

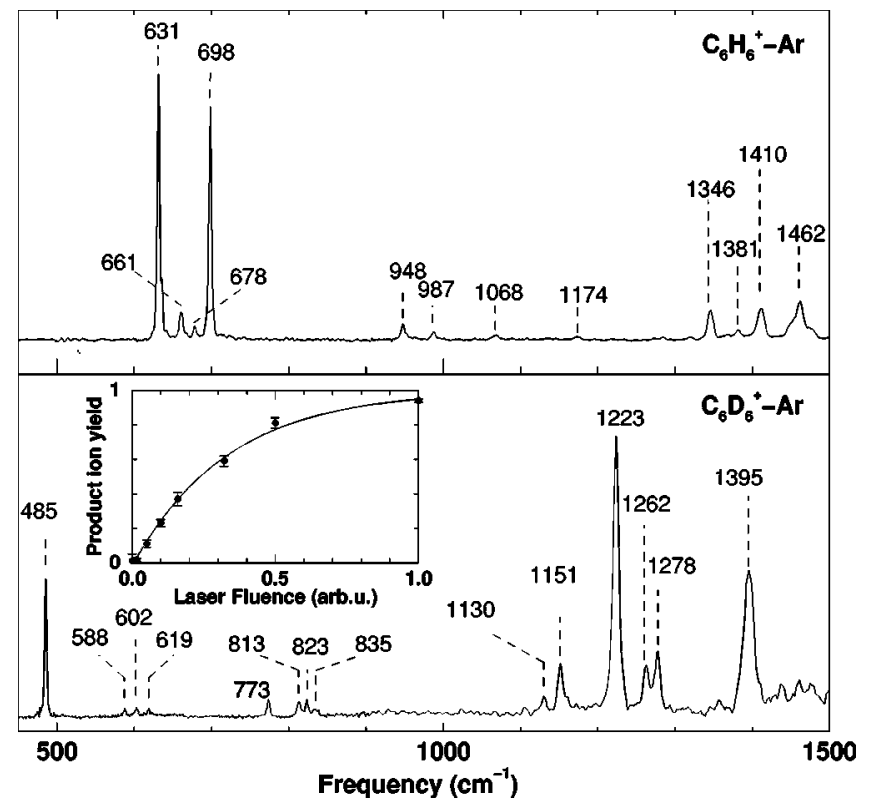

FIG. 2. Observed IR absorption spectrum (linear scale) of the $\mathrm{C}_{6} \mathrm{H}_{6}-\mathrm{Ar}$ cation (upper trace) and of the $\mathrm{C}_{6} \mathrm{D}_{6}-\mathrm{Ar}$ cation (lower trace) with peak positions given in $\mathrm{cm}^{-1}$. In the inset the normalized yield of $\mathrm{C}_{6} \mathrm{D}_{6}^{+}$produced after excitation on the $485 \mathrm{~cm}^{-1}$ mode is shown as a function of IR laser intensity, together with a fit of the data points to a $(1-\exp (-\sigma I))$ dependence.

are of the species $\Gamma_{\mathrm{V}}=2 a_{1 g} \oplus 1 a_{2 g} \oplus 2 b_{1 g} \oplus 1 e_{1 g} \oplus 4 e_{2 g}$ $\oplus 1 a_{2 u} \oplus 2 b_{1 u} \oplus 2 b_{2 u} \oplus 3 e_{1 u} \oplus 2 e_{2 u} \cdot{ }^{19}$ In the electronic ground state of neutral benzene, the $a_{2 u}$ mode and the three $e_{1 u}$ modes are IR active. When the vibrational symmetry is combined with the $E_{1 g}$ electronic symmetry of the ground state of the cation, ${ }^{2}$ the number of possible vibrational modes is doubled. In particular, six ungerade modes of electronicvibrational $A$ symmetry and ten ungerade modes of electronic-vibrational $E$ symmetry result, which are all IR active from the vibrationless level in the ion. Of these, two $A$ modes and two $E$ modes are in the high-frequency $\mathrm{C}-\mathrm{H}(\mathrm{D})$ stretching region, whereas the $E_{1 u}$ component of the $\nu_{16}$ mode, known from ZEKE and MATI experiments to be located at $306 \mathrm{~cm}^{-1}\left(264 \mathrm{~cm}^{-1}\right)$ for the $\mathrm{C}_{6} \mathrm{H}_{6}\left(\mathrm{C}_{6} \mathrm{D}_{6}\right)$ cation, ${ }^{8,11}$ is outside of our scan region at the low frequency side.

In the electronic ground state of the neutral molecule the IR active $a_{2 u} \nu_{11}$ mode [out-of-plane C-H(D) bending] is found at $671 \mathrm{~cm}^{-1}\left(503 \mathrm{~cm}^{-1}\right)$ for $\mathrm{C}_{6} \mathrm{H}_{6}\left(\mathrm{C}_{6} \mathrm{D}_{6}\right) .{ }^{19}$ The strong IR active mode observed at $485 \mathrm{~cm}^{-1}$ in the spectrum of the $\mathrm{C}_{6} \mathrm{D}_{6}-\mathrm{Ar}$ complex is consequently safely assigned to this $\nu_{11}$ mode, which is of electronic-vibrational $E_{1 u}$ symmetry in the $\mathrm{C}_{6} \mathrm{D}_{6}$ cation. This mode has been observed before at $488 \mathrm{~cm}^{-1}$ as a weak peak in ZEKE and MATI spectra, ${ }^{8,11}$ but has never been assigned. The corresponding mode in the $\mathrm{C}_{6} \mathrm{H}_{6}-\mathrm{Ar}$ complex is expected as a single strong peak around $650 \mathrm{~cm}^{-1}$. In the spectrum, however, two almost equally strong peaks are observed at 631 and $698 \mathrm{~cm}^{-1}$ instead, thus forming a textbook example of a Fermi resonance. ${ }^{19}$ To get a Fermi resonance with the $\nu_{11} E_{1 u}$ mode, a nearby combination mode of the same symmetry is required, for which the combination of the linearly Jahn-Teller active $e_{2 g}$ mode $\nu_{6}$ and the quadratically Jahn-Teller active $e_{2 u}$ mode $\nu_{16}$ is the 
only candidate. From a two-mode $\left(\nu_{6}, \nu_{16}\right)$ Jahn-Teller analysis, two components of the $\nu_{6} \nu_{16}$ combination mode with the correct symmetry are expected in this region, so there actually is a three level interaction possible. Assuming that the modes at 631,661 , and $698 \mathrm{~cm}^{-1}$ belong to the Fermi triplet, and taking the same interaction parameter between the $\nu_{11}$ mode and either component of the combination band, the deperturbed frequency of the $\nu_{11}$ mode is found at $665 \mathrm{~cm}^{-1}$, whereas the two $E_{1 u}$ components of the $\nu_{6} \nu_{16}$ combination mode are found at 656 and $669 \mathrm{~cm}^{-1}$. The interaction parameter that is found to describe the observed spectral structure correctly is $23 \mathrm{~cm}^{-1}$.

It is interesting to note that all three components of the Fermi triplet have also been observed in ZEKE and MATI spectroscopy on $\mathrm{C}_{6} \mathrm{H}_{6}$ at the same (within $2 \mathrm{~cm}^{-1}$ ) frequency positions. ${ }^{8,11}$ Moreover, the almost completely mixed outer two components from this triplet indeed appear with the same intensity in these spectra. The two lowest frequency components of this triplet had been assigned to the two doubly degenerate components of the $\nu_{6} \nu_{16}$ combination band, ${ }^{8,11}$ but it was already noted that these modes would then be significantly shifted away from their expected frequencies. $^{11}$ The highest frequency component of the Fermi triplet had not been assigned. The deperturbed values we find for the frequencies of the two components of the $\nu_{6} \nu_{16}$ combination band match the expected frequencies ${ }^{11}$ very well, and provide support to the two-mode Jahn-Teller model as presented by Goode, Hofstein, and Johnson. ${ }^{11}$

Knowing that this Fermi interaction is in effect in the $\mathrm{C}_{6} \mathrm{H}_{6}$ cation, it also has to be present in the deuterated species. Due to a much larger frequency difference between the interacting levels, the effect of the interaction is less pronounced, though it nevertheless gives some IR intensity to the $\nu_{6} \nu_{16}$ combination bands seen at 602 and $619 \mathrm{~cm}^{-1}$. A similar deperturbation analysis on the triplet of modes observed at 485,602 , and $619 \mathrm{~cm}^{-1}$ yields deperturbed frequencies for the $\nu_{11}$ mode of $494 \mathrm{~cm}^{-1}$ and for the $E_{1 u}$ components of the $\nu_{6} \nu_{16}$ combination mode of 598 and $615 \mathrm{~cm}^{-1}$, again in excellent agreement with calculations. ${ }^{11}$ An interaction parameter of $25 \mathrm{~cm}^{-1}$ is found to describe the observations most accurately.

There is another weak peak observed at $678 \mathrm{~cm}^{-1}$ in the spectrum of the $\mathrm{C}_{6} \mathrm{H}_{6}-\mathrm{Ar}$ cation. This peak coincides exactly with the $E_{1 g}$ component of the Jahn-Teller active mode $\nu_{6}$ in the $\mathrm{C}_{6} \mathrm{H}_{6}$ cation, ${ }^{7,8,11}$ which might show up weakly in our spectrum due to the breaking of gerade-ungerade symmetry in the complex. Other possible low frequency ungerade modes of $A$ and $E$ symmetry that this feature could be assigned to are the components of the combination mode of the $b_{1 g}$ mode $\nu_{4}$ at $418 \mathrm{~cm}^{-1}\left(344 \mathrm{~cm}^{-1} \text { for } \mathrm{C}_{6} \mathrm{D}_{6}^{+}\right)^{8,11}$ with the $\nu_{16}$ mode, but these are expected to be above $700 \mathrm{~cm}^{-1}$ for $\mathrm{C}_{6} \mathrm{H}_{6}^{+}$. The peak observed at $588 \mathrm{~cm}^{-1}$ in the spectrum of the deuterated complex might be attributed to the lowest frequency component of this $\nu_{4} \nu_{16}$ combination band, expected to be around $592 \mathrm{~cm}^{-1}$.

For the higher frequency peaks in either of the observed spectra assignment is only possible when a comparison can be made to calculated spectra. Preliminary calculations using the Becke3LYP method with Dunning's D95 $(d, p)$ basis set as implemented in GAUSSIAN 98, and assuming a compressed $D_{2 h}$ distorted structure for the cation, have already yielded remarkable agreement with the observed IR spectra. However, high quality calculated IR spectra for the benzene cation in $D_{6 h}$ symmetry are required to make a meaningful comparison. As both frequency positions and line intensity information are available from the IR absorption spectra shown here, it is expected that it will be possible at that point to assign many of the ungerade fundamental modes in the cation unambiguously, even though the possible occurrence of Fermi interactions is likely to complicate the spectra throughout.

For the strong IR resonance at $485 \mathrm{~cm}^{-1}$ in the $\mathrm{C}_{6} \mathrm{D}_{6}-\mathrm{Ar}$ cation, the absolute value for the fraction of IRlaser-induced dissociation products is measured as a function of IR-laser intensity, and the results are shown in the inset to Fig. 2. If absorption of a single IR photon leads to the appearance of benzene ion dissociation products, the benzene ion branching ratio will have a $(1-\exp (-\sigma I))$ dependence on the IR-laser intensity $I$, with $\sigma$ the absorption cross section for the corresponding single photon transition. In particular, for low values of the product $\sigma I$ the yield of benzene ions will depend linearly on the IR-laser intensity. If the absorption of more than one IR photon is required to overcome the dissociation limit, a higher-than-linear intensity dependence is expected for low values of $\sigma I$. From the measured fractional dissociation curve it is concluded that the vibrational level at $485 \mathrm{~cm}^{-1}$ is located above the dissociation limit of the ionic $\mathrm{C}_{6} \mathrm{D}_{6}-\mathrm{Ar}$ complex. This information combined with the $169 \mathrm{~cm}^{-1}$ redshift of the ionization potential of deuterated benzene upon complexation with an $\mathrm{Ar}$ atom $^{12}$ puts an upper limit on the value of the dissociation limit $D_{0}$ of neutral $\mathrm{C}_{6} \mathrm{D}_{6}-\mathrm{Ar}$ in its electronic ground state of $D_{0}<316 \mathrm{~cm}^{-1}$. This is a more stringent upper limit than the presently accepted experimental upper limit of $340 \mathrm{~cm}^{-1}, 10$ and still some $15 \mathrm{~cm}^{-1}$ below the value of $D_{0}$ found in recent highly accurate $a b$ initio calculations on the ground state of the benzene-Ar complex. ${ }^{20,21}$

We gratefully acknowledge support by the "Stichting voor Fundamenteel Onderzoek der Materie (FOM)" in providing the required beam time on FELIX and highly appreciate the skillful assistance by the FELIX staff. This research is part of the research program of FOM and is in part financially supported by the Council for Chemical Sciences, both of which are financially supported by the "Nederlandse Organisatie voor Wetenschappelijk Onderzoek (NWO),', and receives direct support by the NWO via PIONIER Grant No. 030-66-89.

\footnotetext{
${ }^{1}$ H. A. Jahn and E. Teller, Proc. R. Soc. London, Ser. A 161, 220 (1937). ${ }^{2}$ K. Raghavachari, R. C. Haddon, T. A. Miller, and V. E. Bondybey, J. Chem. Phys. 79, 1387 (1983)

${ }^{3}$ T. A. Miller and V. E. Bondybey, in Molecular Ions: Spectroscopy, Structure and Chemistry, edited by T. A. Miller and V. E. Bondybey (NorthHolland, Amsterdam, 1983), pp. 201-229.

${ }^{4}$ T. A. Barckholtz and T. A. Miller, Int. Rev. Phys. Chem. 17, 435 (1998).

${ }^{5}$ S. R. Long, J. T. Meek, and J. P. Reilly, J. Chem. Phys. 79, 3206 (1983).

${ }^{6}$ I. Fischer, R. Lindner, and K. Müller-Dethlefs, J. Chem. Soc., Faraday Trans. 90, 2425 (1994).

${ }^{7}$ R. Lindner, K. Müller-Dethlefs, E. Wedum, K. Haber, and E. R. Grant, Science 271, 1698 (1996).
} 
${ }^{8}$ R. Lindner, Ph.D. thesis, Technical University of Munich, 1996.

${ }^{9}$ H. Krause and H. J. Neusser, J. Chem. Phys. 97, 5923 (1992).

${ }^{10}$ H. Krause and H. J. Neusser, J. Chem. Phys. 99, 6278 (1993).

${ }^{11}$ J. G. Goode, J. D. Hofstein, and P. M. Johnson, J. Chem. Phys. 107, 1703 (1997).

${ }^{12}$ K. Siglow, R. Neuhauser, and H. J. Neusser, J. Chem. Phys. 110, 5589 (1999).

${ }^{13}$ J. A. Piest, G. von Helden, and G. Meijer, J. Chem. Phys. 110, 2010 (1999).

${ }^{14}$ J. A. Piest, G. von Helden, and G. Meijer, Astrophys. J. 520, L75 (1999).

${ }^{15}$ K. Remmers, R. G. Satink, G. von Helden, J. A. Piest, G. Meijer, and W.

L. Meerts, Chem. Phys. Lett. (in press).
${ }^{16}$ D. Oepts, A. F. G. van der Meer, and P. W. van Amersfoort, Infrared Phys. Technol. 36, 297 (1995).

${ }^{17}$ Th. Weber, A. von Bargen, E. Riedle, and H. J. Neusser, J. Chem. Phys. 92, 90 (1990).

${ }^{18}$ H. Krause and H. J. Neusser, Chem. Phys. Lett. 213, 603 (1993).

${ }^{19}$ G. Herzberg, Molecular Spectra and Molecular Structure (van Nostrand Reinhold, New York, 1945), Pts. I-III.

${ }^{20}$ H. Koch, B. Fernńdez, and O. Christiansen, J. Chem. Phys. 108, 2784 (1998).

${ }^{21}$ H. Koch, B. Fernández, and J. Makarewicz, J. Chem. Phys. 111, 198 (1999). 